\title{
Atypical Femoral Fractures (AFF) from Bone Remodeling Agents in Patients with Cancer
}

\author{
MARIA KORDONI ${ }^{1}$, GEORGIOS RIGAKOS ${ }^{1}$, YOUNG HUN KIM ${ }^{2}$, \\ LOUKAS KAKLAMANIS ${ }^{3}$, OURANIA NIKOLATOU-GALITIS ${ }^{4}$, \\ LINA HADJIYASSEMI ${ }^{1}$, STEFANOS LABROPOULOS ${ }^{1}$ and EVANGELIA RAZIS ${ }^{1}$ \\ ${ }^{1} 3 r d$ Department of Oncology, Hygeia Hospital, Athens, Greece; \\ ${ }^{2}$ Neuroscience Department, John Hopkins University, Baltimore, MD, U.S.A.; \\ ${ }^{3}$ Onassis Cardiac Surgery Center, Athens, Greece; \\ ${ }^{4}$ School of Dentistry, National and Kapodistrian University of Athens, Athens, Greece
}

\begin{abstract}
Anti-resorptive drugs like bisphosphonates (BP) and denosumab are widely used for prevention and treatment of skeletal diseases, such as osteoporosis and bone metastases and mainly work through the prevention of osteoclast-mediated bones resorption. BP can lead to atypical femoral fractures ( $A F F$ ) that is a rare, easily misdiagnosed treatment-related complication with great impact on the quality of life of patients. We present a concise review of the literature on $B P$ related AFF based on the cases of 3 breast cancer patients that reflect the diagnostic pitfall of this rare entity. In conclusion, breast cancer patients very often are exposed to BP use and are at risk of developing AFF.
\end{abstract}

Bisphosphonate therapy is one of the principal methods of treatment for skeletal diseases, such as osteoporosis and bone metastases. These anti-resorptive drugs, including alendronate and zoledronate, work to hinder bone loss and thereby the occurrence of classic fractures through the prevention of osteoclast-mediated bone resorption. Bisphosphonate (BP) use can lead to adverse effects, including osteonecrosis of the jaw (ONJ) and atypical femoral fractures (AFF) (1).

Breast cancer patients with bone metastases receive BPs to prevent skeletal complications, and often undergo radiation therapy (RT) or surgery to treat such complications (2). An accurate diagnosis of treatment-related side-effects

Correspondence to: Dr. Georgios Rigakos, 3rd Oncology Department, Hygeia Hospital, 4, Erythrou Stavrou \& Kifisias Av. Marousi, 15123 Greece. Tel: +30 6947948628/+30 2106867165, e-mail: grigakos@oncologists.gr

Key Words: AFF, atypical femoral fracture, antiresorptive, bisphosphonates, cancer. is critical, especially in the case of AFFs, as inappropriate treatment can result in serious consequences.

AFFs are uncommon insufficiency fractures of the subtrochanteric region that occur without a high-energy event. These fractures develop in bones that are abnormal in mineralization and elasticity. This abnormality is thought to be a result of the accumulation of micro-damages after interference with normal bone remodeling and repair, which is the principal mechanism of function of BPs $(3,4)$. Pain in the area of an impending AFF may often be mistaken as a symptom of a bone metastasis and may be treated with RT which can worsen the condition.

Due to the increasing number of AFF cases, there has been research on the causal link between bisphosphonates and atypical femoral fractures over the recent years. Although AFFs are now recognized as a potential complication in patients receiving BP therapy for osteoporosis, this correlation has not been discussed to the same extent in patients receiving BP for bone metastases (5). In both cases this drug-related adverse event occurs mainly in women (6). In this report, we present three cases of atypical femoral fractures in female patients that had been diagnosed with metastatic breast cancer and treated with bisphosphonates, in the hope to further support the link between AFFs and BP treatment for cancer patients and highlight the specific concerns in this setting.

\section{Case 1}

A 68-year-old woman underwent quadrantectomy of the right breast and axillary lymph node (LN) dissection in May 2006 for breast cancer staged as T3N2M0, negative for estrogen (ER) and progesterone (PR) receptors while positive for HER2 overexpression. She received adjuvant chemotherapy involving 4 cycles of cyclophosphamide/epirubicin/5-FU followed by 4 cycles of docetaxel, RT and trastuzumab every 3 weeks until November 2007. 
In December 2007, she relapsed in the breast, bone, soft tissue, and lung and received chemotherapy with paclitaxel/carboplatin/trastuzumab and was also started on BPs. Several regimens for relapses followed, including capecitabine with lapatinib, vinorelbine/trastuzumab, gemcitabine with carboplatin/ trastuzumab and liposomal doxorubicin/trastuzumab, trastuzumab emtansine, eribulin with trastuzumab, always with zoledronate.

In February 2014, she had pain in the left leg and difficulty in walking, but a magnetic resonance imaging (MRI) of the pelvis and hips showed only a previously identified metastasis in the left sacroiliac area, that remained unchanged. An MRI of the lumbar spine and an electromyography (EMG) were not helpful and a computed positron-emission tomography/tomography (PET/CT) did not show uptake in the left femur. However, an X-ray of the area was somewhat suspicious, due to a focal thickening of the periosteum of the upper third of the left femur. At this point the pain was much worse and RT was considered, but it was decided to wait and see if the pain responded to the new regimen of eribulin and trastuzumab. Meanwhile, the patient developed osteonecrosis of the jaw (ONJ) in March 2014 and BPs were discontinued. A bone scan in May 2014 identified a new lesion in the left femoral neck and an MRI in late July showed a $3.5 \mathrm{~cm}$ lesion in the left femoral diaphysis, which was barely visible before. The orthopedist recommended RT to the area, so she received 45 Gy to the left femur and sacral area and she was switched to pertuzumab/nabpaclitaxel/trastuzumab. After 3 months, pain in the left hip persisted and the patient required a cane to walk. Follow-up imaging showed improvement in her systemic disease but also an increase in size in the focus of the left femur and abnormal appearance of surrounding tissues, attributed to RT.

In November 2014, the patient sustained a traumatic left femoral neck fracture (Figure 1) and underwent intramedullary nailing. The bone biopsy did not show malignant cells but could not be highly diagnostic of atypical lesions as the material was obtained from the intramedullary space and had scarce cellular elements.

Her CT scans in January 2015 showed stable disease. She continued maintenance treatment with pertuzumab and trastuzumab. The pain in the hip persisted and it was obvious by then that she had sustained a fracture in the area of a BP related lesion, which had been misdiagnosed as a metastasis and treated with RT. The fracture never healed and several options were entertained but finally she was submitted to total hip replacement in November 2015.

The pathology report showed extensive necrosis of the bone (Figure 2). She recovered well after the operation and continued on the same regimen until December 2016. At the time the patient was diagnosed with leptomeningeal carcinomatosis and eventually deteriorated and passed away in July 2017.

\section{Case 2}

A 55-year-old woman had a quadrantectomy of the right breast and axillary lymph node dissection for an early breast cancer in 2005. Her tumor was negative for ER and PR while positive for HER2 overexpression, like the previous patient. She underwent chemotherapy with 4 cycles of cyclophosphamide/epirubicin, 4 cycles of paclitaxel/ trastuzumab and radiotherapy with trastuzumab for one year. She relapsed with bone metastases in 2010 and was treated with capecitabine, trastuzumab and zoledronate until July 2011, and continued with zoledronate and trastuzumab until July 2013 when zoledronate was switched to denosumab. In March 2014 she started capecitabine again due to new bone lesions until October 2014, when she went back on maintenance with trastuzumab and denosumab. In January 2015 she had enlarged mediastinal LN and a new bone lesion, so vinorelbine was added. In April she presented with severe pain in the right hip and difficulty in walking. Imaging was interpreted as metastatic disease by the orthopedist and he suggested RT.

Meanwhile she was switched to trastuzumab emtansine (TDM1) as new supraclavicular lymph nodes had appeared and denosumab was continued. An MRI in 10/2015 showed lesions in the right hip and in the femoral diaphysis that were interpreted as metastatic, so she had RT to the area (30 Gy) in November 2015. Unfortunately, though the hip pain subsided, the femur continued to hurt and the x-ray was diagnostic of an AFF with a linear fracture amidst a focal periosteal thickening (Figure 3). Denosumab was discontinued and she underwent hip replacement in January 2016. Pathology was consistent with necrotic bone without malignant cells. She recovered well and continued on TDM1. In June 2016 she also had a fracture in the 5th left metatarsal bone and in September 2016 she was started on lapatinib and trastuzumab, due to disease progression, until November 2017 when she had a nodal recurrence and was referred for local RT while continuing with the same regimen.

\section{Case 3}

A 66-year-old woman was diagnosed with T1N1 ER/PR negative, HER2 positive breast cancer in 2003 and was treated with modified radical mastectomy, and adjuvant sequential chemotherapy with epirubicin paclitaxel and cyclophosphamide methotrexate fluorouracil (CMF). She relapsed in bone in 2008 and was treated with zoledronate, liposomal doxorubicin and trastuzumab.

She continued with maintenance treatment with trastuzumab and zoledronate until 10/2010 when her therapy was changed to capecitabine and lapatinib for disease progression in iliac lymph nodes, followed by maintenance treatment with lapatinib and zoledronate. Disease 


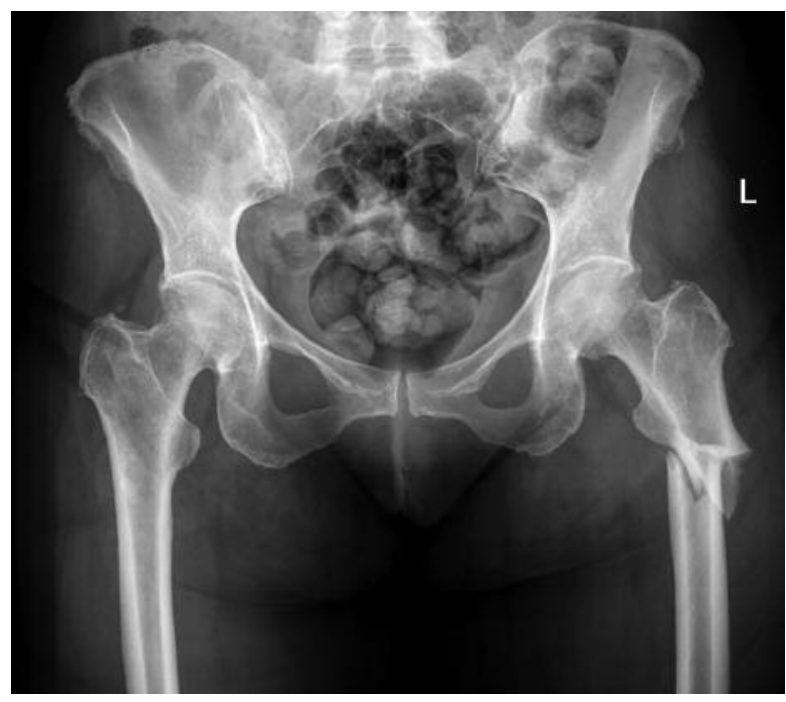

Figure 1. X-ray of left femur with fracture following fall (patient 1).

progression in bone was documented two years later and she was placed on trastuzumab with lapatinib and zoledronate, and in January 2013 she was placed on TDM1 and zoledronate for a disease progression in lymph nodes.

In December 2014 she developed ONJ and the zoledronate was discontinued. In June 2015 she complained of pain and swelling of the left lower extremity and the x-ray was suspicious for an early bisphosphonate related lesion without fracture. The zoledronate had been discontinued so we decided to manage her conservatively with avoidance of weight bearing. MRI and x-ray in September of 2015 confirmed the finding, and she was gradually improving, while systemic disease was still under control by TDM1.

The pain had subsided and she was able to walk without a cane, however in November 2016 she sustained a traumatic fracture and underwent hip replacement at another institution. No tissue specimen was sent for histology. After this the patient continued on trastuzumab and her gait gradually improved. Of note she suffered a recurrence of the ONJ that was managed surgically. Additionally, she had a growing lesion in the left choroid plexus, which was stable on TDM1, but grew on trastuzumab maintenance and was finally treated with radiosurgery in December 2017. The patient is clinically stable and was restarted on TDM1 in November 2017.

\section{Discussion}

As more incidents of AFF have been reported, its diagnosis has become more standardized, as seen in the criteria outlined by the American Society for Bone and Mineral

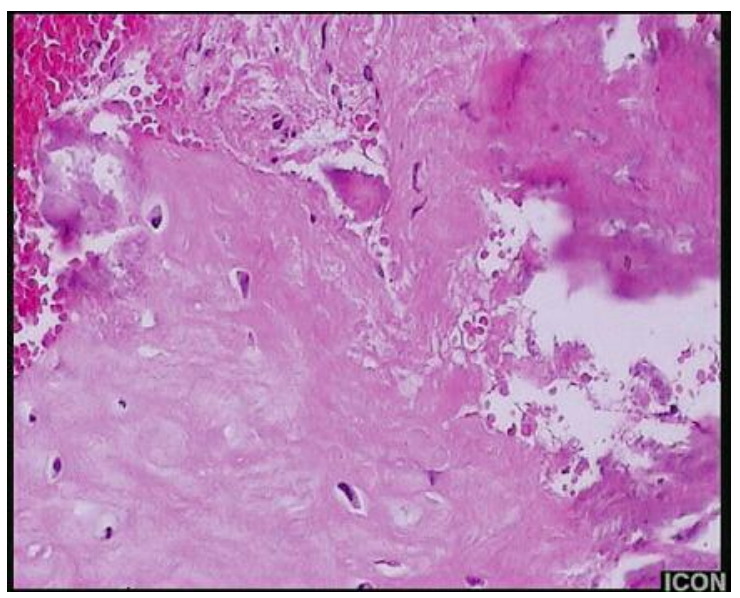

Figure 2. Histology of bone sample following total hip replacement in patient 1 showing extensive necrosis with no evidence of metastasis.

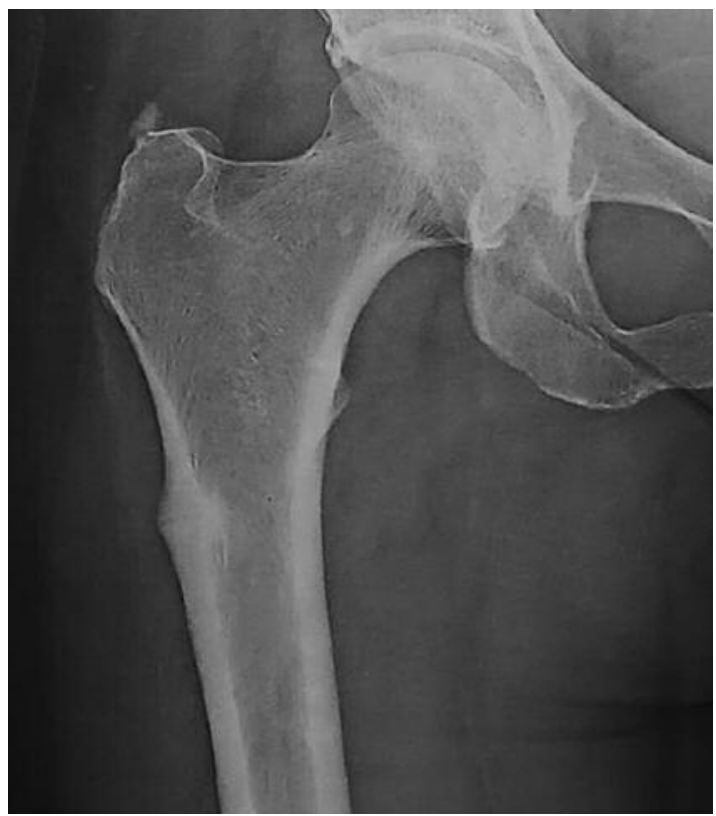

Figure 3. X-ray of right femur (patient 2) with linear fracture in a focus of thickened periosteum, suggestive of atypical femoral fracture.

Research (ASBMR) $(7,8)$ (Table I). AFFs are classified as non-comminuted fractures that take on a transverse or oblique configuration occurring in the subtrochanteric area of the femoral or humeral shaft. They develop spontaneously or due to low trauma, and often occur bilaterally. They may also be accompanied by other symptoms and signs including prodromal pain in the groin or thigh, and cortical thickening (8). 
Table I.

\section{ASBMR criteria}

Four of five major criteria should be observed. Additional minor criteria are not necessary for diagnosis but should be observed in association to the major criteria.

Major

- The fracture is associated with minimal or no trauma, as in a fall from standing height or less.

- The fracture line originates at the lateral cortex and is substantially transverse in its orientation, although it may become oblique as it progresses medially across the femur.

- Complete fractures extend through both cortices and may be associated with a medical spike incomplete fractures involve only the lateral cortex.

- The fracture is non-comminuted or minimal comminuted.

- Localized periosteal or endosteal thickening of the lateral cortex is present at the fracture site ("beaking" or "flaring").

Minor

Generalized increase in cortical thickness of the femoral diaphysis. Unilateral or bilateral prodromal symptoms such as dull or aching pain in the groin or thigh.

Bilateral incomplete or complete femoral diaphysis fractures. Delayed fracture healing.

Evaluation is recommended in patients with new onset of groin or mid-thigh pain who have been taking bisphosphonates for more than 3 years. Conventional radiography is usually the initial imaging procedure and the diagnostic finding is a periosteal and endosteal callus formation (beaking) and a transverse fracture line at the lateral cortex in the subtrochanteric area of the femoral shaft (8). Imaging may include MRI, which could detect a cortical fracture line and associated bone and marrow edema or hyperemia. CT may also show a cortical fracture or lucency and associated new-bone formation. Radionuclide bone scan has less specificity than MRI or CT but may detect focal bone and marrow hyperemia. If cortical lucency is found, the lesion could be considered an incomplete AFF but, if no lucency is seen but marrow edema is present, such lesions are described as stress reaction (8).

Unfortunately, in contrast to the many studies that have associated AFFs with BP therapy for the treatment of osteoporosis, there have been only a few cases that relate AFFs with BP therapy for the treatment of bone metastasis. According to the latest ASBMR task report there had been only one case of subtrochanteric femoral fracture following BP therapy for bone metastasis reported before 2010. This case is especially interesting because the patient was male (4) but the rest of the reported cases were female. Since the initial description of this entity, there have been some reports on cancer patients with AFF receiving high-dose intravenous BP that mainly report small series or single cases (9).

One study analyzed the occurrence of AFFs in patients with skeletal malignancy. From a total of 327 patients, four patients had developed or had impending AFFs. These four patients were all women, three of whom had breast cancer. However, there was no significant difference in the doses or duration of BP treatment between the patients who had AFFs and those who had not (10). Another study examined a total of 62 patients with BP therapy for breast cancer or multiple myeloma. Six cases of AFFs were identified, four of which were in breast cancer patients. Of those six, five had bilateral findings and two developed ONJ as well. In this study however, it was recognized that patients who had developed AFFs had received significantly more BP infusions for a longer period of treatment (5). More recently a study in breast cancer patients described increased rates of periosteal thickening and AFF in patients receiving antiresorptive drugs for bone metastases compared to a control group of patients with breast cancer without bone disease that did not receive any antiresorptive drugs (11). Additionally, in a retrospective study, 23 cases of AFF were identified in a population of $10587 \mathrm{BP}$ users in a period of 10 years and the development of AFF was significantly higher in patients using alendronate compared to other BPs (12). Of note there are also cases that relate AFF to denosumab use $(13,14)$.

Regarding the mechanism of AFF formation, the appearance of them on imaging suggests that damage accumulates within the bone cortex over a long period and that AFFs are stress or insufficiency fractures. Long-term therapy with BPs can give rise to severe inhibition not only of osteoclastic activity but also of osteoblastic activity and a severe suppression of bone turnover. This protracted remodeling suppression leads to compromised bone quality $(15,16)$. At the same time, mineralization of the osteoid tissue continues resulting in increased brittleness of bone that makes it prone to fractures (16). This accumulation of abnormal bone tissue is thought to be the basis of atypical fracture pathogenesis. To our knowledge it has not been related, so far, to the existence of either lytic or blastic bone metastases but it should be noted that abnormal osteoblastic activity occurs in both types of metastases and may contribute to the AFF pathogenesis.

Interestingly despite the increasing number of AFFs and ONJ reported in the literature, simultaneous occurrence of these two complications originally had only been reported in a very small number of patients, mainly after parenteral bisphosphonates in cancer patients $(17,18)$, but lately it has been found that patients with AFF under use of parenteral BPs for malignant disease have up to $30 \%$ chance of developing simultaneous ONJ (19). 
Another part of bone metabolism that is affected under treatment is the formation of new blood vessels and the significant reduction in circulating vascular endothelial growth factor levels. This effect is more prominent in ONJ formation. Combining bisphosphonates with antiangiogenic agents has been suggested to induce ONJ more frequently than using bisphosphonates alone (17). For instance, there have been reports of patients with ONJ caused by antiangiogenic agents such as sunitinib, bevacizumab and everolimus with or without bisphosphonates (20). Substances such as nicotine, alcohol and drugs like glucocorticoids and proton pump inhibitors are also implicated in the development of atypical femoral fractures via weakening of the bone equilibrium (21).

Beside the role of bisphosphonates in the development of AFF, concomitant administration of trastuzumab as an independent risk factor would be worth evaluating. Of note, all three patients described herein had ER negative HER2 positive tumors. Trastuzumab is one of the most widely used agents for the management of metastatic breast cancers with HER2 overexpression. Studies in mice carrying human breast tumors that overexpresses HER2 have shown that trastuzumab induces normalization and regression of the vasculature by modulating the effect of different pro and anti- angiogenic factors $(22,23)$. Maybe that is the reason why it is associated with $\mathrm{ONJ}$ and $\operatorname{AFF}(23,24)$. In addition, prognosis of HER2-positive breast cancer patients has changed since the introduction of trastuzumab with prolonged survival rates (25). It is logical to assume that prolonged survival may also be accompanied by prolonged exposure to drugs inducing AFF like zoledronate along with the anti-HER2 treatment itself, although there have been no reports in the literature on the development of ONJ, solely due to trastuzumab. Patients with estrogen receptor positive breast cancer also have prolonged survival and experience AFFs related to antiresorptive drugs received as treatment for osteoporosis (that may be aggravated by endocrine treatment i.e. aromatase inhibitors) or for metastatic bone disease. Prevalence of AFFs in this population is affected by the differential anabolic effect of endocrine treatment options with women receiving aromatase inhibitors possibly being at higher risk especially taking under consideration that they are in greater need of anti-osteoporosis treatment (26).

There are no controlled studies evaluating medical and surgical treatment protocols in patients with AFF. Management of patients includes fracture fixation, and initiation of medical management. Bisphosphonates should be discontinued and adequate calcium and vit-D supplementation should be given. Orthopedic intervention is necessary for patients with complete fractures and for those with incomplete fractures accompanied by significant pain. Conservative management (limited weight bearing, calcium and vit-D supplementation, observation) may be adequate for patients with incomplete fractures and for those with complete fractures not accompanied by significant pain. Another option in some cases could be the use of parathyroid hormone (PTH), although its use is thought to be contraindicated in patients with preexisting malignancies, unless other drugs have failed and the benefits of PTH outweigh potential risks $(27,28)$.

Stress fractures after radiotherapy are not rare and often pose a diagnostic challenge between stress fractures and metastatic disease. Pelvic stress fractures are well recognized after radiotherapy. Common primary pathologies leading to this are cervical and rectal cancer. The lower limbs are the least known sites for post radiotherapy stress fractures but still, since radiotherapy leads to direct cell death and damage of the microvasculature, when administered to the lower extremities, it could theoretically render the bone vulnerable to stress fractures (21). Radiation causes cellular depletion, extracellular matrix changes and microvascular damage resulting in total tissue hypoxia that further facilitates the formation of an AFF (29). It is therefore of utmost importance to promptly recognize the symptoms and signs that discriminate a possible AFF from a bone metastasis as giving radiotherapy to the former would significantly worsen the outcome, since radiotherapy results in unintended injury to overlying tissues and contributes to poor wound healing.

In conclusion, although the incidence of AFF in patients on bisphosphonates (and other bone remodeling therapy) for skeletal malignancies may be low, there is a pressing need for awareness and further study of the mechanisms by which this condition develops. These fractures usually occur in patients with long survival and have devastating consequences on their quality of life so a proper survivorship plan for patients receiving treatment for cancer related osteoporosis and bone metastases must include awareness to recognize and better avoid this adverse event.

\section{References}

1 Drake MMT, Clarke BL and Khosla S: Bisphosphonates: Mechanism of action and role in clinical practice. Mayo Clin Proc 83(9): 1032-1045, 2008

2 Kim YS and Park WC: Atypical subtrochanteric femur fracture in patient with metastatic breast cancer treated with zoledronic acid. Breast Cancer 15(2): 261-264, 2012.

3 Giannini S, Chiarello E, Tedesco G, Cadossi M, Luciani D, Mazzotti A and Donati DM: Atypical femoral fractures. Clin Cases Miner Bone Metab 10(1): 30-33, 2013.

4 Bush LA and Chew FS: Subtrochanteric femoral insufficiency fracture following bisphosphonate therapy for osseous metastases. Radiol Case Rep 3(4): 232, 2008.

5 Chang ST1, Tenforde AS, Grimsrud CD, O'Ryan FS, Gonzoledronatelez JR, Baer DM, Chandra M and Lo JC: Atypical femur fractures among breast cancer and multiple myeloma patients receiving intravenous bisphosphonate therapy. Bone 51(3): 524-527, 2012. 
6 Kharazmi M, Hallberg P and Michaëlsson K: Gender related difference in the risk of bisphosphonate associated atypical femoral fracture and osteonecrosis of the jaw. Ann Rheum Dis 73: 1594, 2014.

7 Shane E, Burr D, Ebeling PR, Abrahamsen B, Adler RA, Brown TD, Cheung AM, Cosman F, Curtis JR, Dell R, Dempster D, Einhorn TA, Genant HK, Geusens P, Klaushofer K, Koval K, Lane JM, McKiernan F, McKinney R, Ng A, Nieves J, O'Keefe R, Papapoulos S, Sen HT, van der Meulen MC, Weinstein RS and Whyte M; American Society for Bone and Mineral Research: Atypical subtrochanteric and diaphyseal femoral fractures: Report of a task force of the American Society for Bone and Mineral Research. J Bone Miner Res 25: 2267-2294, 2010.

8 Shane E, Burr D, Abrahamsen B, Adler RA, Brown TD, Cheung AM, Cosman F, Curtis JR, Dell R, Dempster DW, Ebeling PR, Einhorn TA, Genant HK, Geusens P, Klaushofer K, Lane JM, McKiernan F, McKinney R, Ng A, Nieves J, O'Keefe R, Papapoulos S, Howe TS, van der Meulen MC, Weinstein RS and Whyte MP: Atypical Subtrochanteric and Diaphyseal Femoral Fractures: Second Report of a Task Force of the American Society for Bone and Mineral Research. J Bone Miner Res 29: 1-23, 2014.

9 Hayashi K1, Aono M, Shintani K and Kazuki K: Bisphosphonaterelated atypical femoral fracture with bone metastasis of breast cancer: case report and review. Anticancer Res 34(3): 1245-1249, 2014.

10 Puhaindran ME1, Farooki A, Steensma MR, Hameed M, Healey JH and Boland PJ: Atypical subtrochanteric femoral fractures in patients with skeletal malignant involvement treated with intravenous bisphosphonates. J Bone Joint Surg Am 93(13): 1235-1242, 2011.

11 Ota S, Inoue R, Shiozaki T, Yamamoto Y, Hashimoto N, Takeda O, Yoshikawa K, Ito J and Ishibashi Y: Atypical femoral fracture after receiving antiresorptive drugs in breast cancer patients with bone metastasis. Breast Cancer (Tokyo, Japan) 24(4): 601-607, 2017.

12 Edwards BJ, Sun M, West DP, Guindani M, Lin YH, Lu H, Hu M, Barcenas C, Bird J, Feng C, Saraykar S, Tripathy D, Hortobagyi GN, Gagel R and Murphy WA Jr.: incidence of atypical femur fractures in cancer patients: The MD Anderson Cancer Center Experience. J Bone Miner Res 31(8): 1569-1576, 2016.

13 Khow KS and Yong TY: Typical femoral fracture in a patient treated with denosumab. J Bone Miner Metab 33(3): 355-358, 2015.

14 Yang SP, Kim TW, Boland PJ and Farooki A: Retrospective review of atypical femoral fracture in metastatic bone disease patients receiving denosumab therapy. Oncologist 22(4): 438444, 2017.

15 Mashiba T, Hirano T, Turner C, Forwood M, Johnston C and Burr D: Suppressed bone turnover by bisphosphonates increases microdamage accumulation and reduces some biomechanical properties in dog rib. J Bone Miner Res 15: 613-620, 2000.

16 Ettinger B, Burr DB and Ritchie RO: Proposed pathogenesis for atypical femoral fractures: lessons from materials research. Bone 55(2): 495-500, 2013.

17 Sánchez A and Blanco R: Osteonecrosis of the jaw (ONJ) and atypical femoral fracture (AFF) in an osteoporotic patient chronically treated with bisphosphonates. Osteoporos Int 28(3): 1145-1147, 2017.
18 Afif H, Mukka S, Sjödén G and Sayed-Noor AS: Do bisphosphonate-related atypical femoral fractures and osteonecrosis of the jaw affect the same group of patients? A Pilot Study. Orthopedic Rev 6(1): 5067, 2014.

19 Graves LL, Bukata SV, Aghazadehsanai N, Chang TI, Garrett NR and Friedlander AH: Patients receiving parenteral bisphosphonates for malignant disease and having developed an atypical femoral fracture are at risk of concomitant osteonecrosis of the jaw: An evidence-based review. J Oral Maxillofac Surg 74(12): 2403-2408, 2016.

20 Rosella D, Papi P, Giardino R, Cicalini E, Piccoli L and Pompa G: Medication-related osteonecrosis of the jaw: Clinical and practical guidelines. J Int Soc Prev 6(2): 97-104, 2016.

21 Tins BJ, Garton M, Cassar-Pullicino VN, Tyrrell PNM, Lalam $\mathrm{R}$ and Singh J: Stress fracture of the pelvis and lower limbs including atypical femoral fractures - a review. Insights Imaging 6(1): 97-110, 2015.

22 Izumi Y, Xu L, di Tomaso E, Fukumura D and Jain RK: Tumour biology: herceptin acts as an anti-angiogenic cocktail. Nature 416(6878): 279-280, 2002.

23 Wen XF1, Yang G, Mao W, Thornton A, Liu J, Bast RC Jr. and Le XF: HER2 signaling modulates the equilibrium between proand antiangiogenic factors via distinct pathways: implications for HER2-targeted antibody therapy. Oncogene 25(52): 69866996, 2006.

24 Pilanci KN, Alco G and Ordu C: Is administration of trastuzumab an independent risk factor for developing osteonecrosis of the jaw among metastatic breast cancer patients under zoledronic acid treatment? Medicine (Baltimore) 94(18): e671, 2015

25 Kast K, Schoffer O and Link T: Trastuzumab and survival of patients with metastatic breast cancer. Arch Gynecol Obstet 296(2): 303-312, 2017.

26 Chau S, Chandra M, Grimsrud CD, Gonzalez JR, Hui RL and Lo JC: Femur fracture classification in women with a history of breast cancer. J Bone Oncol 3(2): 49-53, 2014.

27 Chiang CY, Zebaze RM, Ghasem-Zadeh A, Iuliano-Burns S, Hardidge A and Seeman E: Teriparatide improves bone quality and healing of atypical femoral fractures associated with bisphosphonate therapy. Bone 52: 360-365, 2013.

28 Watanabe A, Yoneyama S, Nakajima M, Sato N, Takao-Kawabata R, Isogai Y, Sakurai-Tanikawa A, Higuchi K, Shimoi A, Yamatoya $\mathrm{H}$, Yoshida K and Kohira T: Osteosarcoma in Sprague-Dawley rats after long-term treatment with teriparatide (human parathyroid hormone (1-34)). J Toxicol Sci 37: 617-629, 2012.

29 Jacobson KL, Johnson BM, Dedhia DR, Niknam-Bienia S and Wong KA: Impaired wound healing after radiation therapy: A systematic review of pathogenesis and treatment. JPRAS Open 13: 92-105, 2017. 